Setting a new standard in the analysis of binary stars

Editors : will be set by the publisher

EAS Publications Series, Vol. ?, 2018

\title{
PROBING THE MODELS: ABUNDANCES FOR HIGH-MASS STARS IN BINARIES
}

\author{
K. Pavlovski ${ }^{1}$ and J. Southworth ${ }^{2}$
}

\begin{abstract}
The complexity of composite spectra of close binary star system makes study of the spectra of their component stars extremely difficult. For this reason there exists very little information on the photospheric chemical composition of stars in close binaries, despite its importance for informing our understanding of the evolutionary processes of stars. In a long-term observational project we aim to fill this gap with systematic abundance studies for the variety of binary systems. The core of our analysis is the spectral disentangling technique, which allows isolation of the individual component star spectra from the time-series of observed spectra.

We present new results for high-mass stars in close binaries. So far, we have measured detailed abundances for 22 stars in a dozen detached binary systems. The parameter space for the stars in our sample comprises masses in the range $8-22 \mathrm{M}_{\odot}$, surface gravities of 3.14.2 (c.g.s.) and projected rotational velocities of $30-240 \mathrm{~km} \mathrm{~s}^{-1}$. Whilst recent evolutionary models for rotating single stars predict changes in photospheric abundances even during the main sequence lifetime, no star in our sample shows signs of these predicted changes. It is clear that other effects prevail in the chemical evolution of components in binary stars even at the beginning of their evolution.
\end{abstract}

\section{Motivation}

Detached eclipsing binary stars (dEBs) are the primary source of fundamental stellar quantities: mass $M$ and radius $R$. Both components must be spectroscopically detectable for direct determination of these quantities. Modern astronomical instrumentation and analytical methods enable a high accuracy and precision to be reached, reducing errors in the masses and radii for the stars in binaries to $1 \%$ or less (e.g. Pilecki et al. 2013). A critical examination of published analyses found

\footnotetext{
${ }^{1}$ Department of Physics, Faculty of Science, University of Zagreb, 10000 Zagreb, Croatia

2 Astrophysics Group, Keele University, Staffordshire ST5 5BG, UK
} 
Setting a new standard in the analysis of binary stars

95 systems satisfying the criterion of $3 \%$ in the uncertainties for $M$ and $R$ (Torres et al. 2010). The DEBCat catalogue lists 159 systems 1 .

For a useful comparison with theoretical evolutionary models further observables are needed: effective temperature, $T_{\text {eff }}$, and metallicity, $[\mathrm{M} / \mathrm{H}]$. Only the ratio of the $T_{\text {eff }} \mathrm{S}$ of the two stars is provided by the light curve analysis. The $T_{\text {eff }}$ of one component must be determined from other sources, e.g. spectral energy distribution, colour indices, or spectral type estimates. Far worse is the situation with empirical determination of the components' metallicity. Of the 95 systems listed by Torres et al. (2010), fewer than half have an empirically estimated metallicity. Detailed abundance studies has been performed for only four systems, including two from our own work (see Sect. 5.).

The method of Doppler tomography (Bagnuolo \& Gies 1991), and its generalisation as spectral disentangling (Simon \& Sturm 1994, Hadrava 1995) enables the study of the individual spectra of the components of binary star systems. This is particularly useful for the determination of $T_{\text {eff }}$ and metallicity (c.f. Pavlovski \& Southworth 2012, and references therein). Since the structure and evolution of a star depends on its chemical composition, the degeneracy in the location of a star in evolutionary diagrams can only be broken if an empirical metallicity is known.

\section{Theoretical framework}

In the last two decades a new generation of stellar evolutionary models has been developed (c.f. Maeder \& Meynet 2012, Langer 2012). The inclusion of stellar rotation has had profound effects on these models (Meynet \& Maeder 2000, Heger \& Langer 2000, Heger et al. 2000). Centrifugal force changes the stellar shape and structure, causes meridional circulation and induces turbulent mixing. In turn, the $T_{\text {eff }}$ and luminosity of the rotating star changes, affecting its liftime. As a consequence, the surface abundance pattern changes too, heavily affecting the expected photospheric nitrogen abundance.

The calculations predict an increase in nitrogen abundance with increasing stellar mass, increasing initial rotational velocity, and decreasing metallicity. This makes nitrogen abundance viable as a new observable (Köhler et al. 2012). However, caution is needed since Hunter et al. (2009) have found from the observations of a large sample of the Galactic and Magellanic Clouds B stars the relation between nitrogen enhancement and stellar parameters (mass and projected rotational velocity, $v \sin i$ ) to be more complex than predicted from the models. Subsequently, Morel et al. (2008) did not find any tight correlation of the nitrogen abundance with the strength of the magnetic field. This was also supported by extension of the analysis to O stars (Martins et al. 2012).

Recently, comprehensive grids of theoretical stellar evolutionary models incorporating rotation have been available by the Utrecht (Brott et al. 2011) and Geneva (Ekström et al. 2012) groups. These models differ in the treatment of

\footnotetext{
${ }^{1}$ http://www.astro.keele.ac.uk/jkt/debcat/
} 
rotational mixing, as well as in the calibration. Brott et al. (2011) tailored their grids of different initial composition to match the results of VLT-FLAMES survey of massive stars in the Magellanic Clouds and the Galaxy (Evans et al. 2005). In consequence, their models have no scaled-solar composition, and result in a rather low metallicity for Galactic stars, $Z=0.088$. In contrast, Ekström et al. (2012) use scaled-solar compositions, and galactic models are represented with the metallicity $\mathrm{Z}=0.014$.

\section{Spectral disentangling}

The complexity of composite spectra of close binaries makes the study of the individual stellar spectra extremely difficult. Shifts of the spectral lines due to the orbital motion of components in binary or multiple systems are essential for the determination of stellar masses. Spectral lines overlap for much of each orbital cycle, and the secondary star may be much fainter than the primary, and thus contribute only a small fraction of the total light of the system. To these problems should be added the intrinsic broadening of spectral lines in OB stars, and high $v \sin i$ in binaries. All these pose a difficulty for accurate measurements of radial velocities (RVs) for the components, lowering the accuracy of the derived stellar masses (c.f. Andersen 1991, Torres et al. 2010) or making the fainter star impossible to detect. Since the first spectroscopic detection of binary stars in the last decades of the 19th century, binary star spectra have been used almost solely for RV measurement. Important astrophysical information contained in these spectra was ignored due to the inability of researchers to extract individual spectra of the components.

The method of spectral disentangling (SPD) is the way to avoid these obstacles. Bagnuolo \& Gies (1992) successfully applied the method of Doppler tomography and isolated component star spectra for the case when the RVs of the individual exposures were already known. Simon \& Sturm (1994) generalised tomographic separation by setting a matrix equation from a time-series of binary star spectra. A set of linear equations is solved directly for the orbital elements and the component spectra, thus bypassing the determination of RVs entirely. An independent formulation of the equations of SPD in the Fourier space by Hadrava (1995) has been particularly advantageous since the calculations are more efficient and computationally less demanding. An overview of SPD and its variants is given by Pavlovski \& Hensberge (2010).

In comprehensive studies of V578 Mon, Hensberge et al. (2000) and Pavlovski \& Hensberge (2005) constructed a complete procedure for the analysis of spectroscopically double-lined eclipsing binary stars. In the core of the procedure is SPD, which yields the optimal set of orbital elements and isolated individual spectra of the components. Further, the analysis of these spectra provides the atmospheric parameters for the components: $T_{\text {eff }} \mathrm{s}$, metallicity, detailed abundances and $v \sin i$ values. In turn, the atmospheric parameters are fed back into fine-tuning the light curve solution. In an iterative cycle an optimal set of stellar and binary parameters can be achieved. Further development and applications are given in Pavlovski 


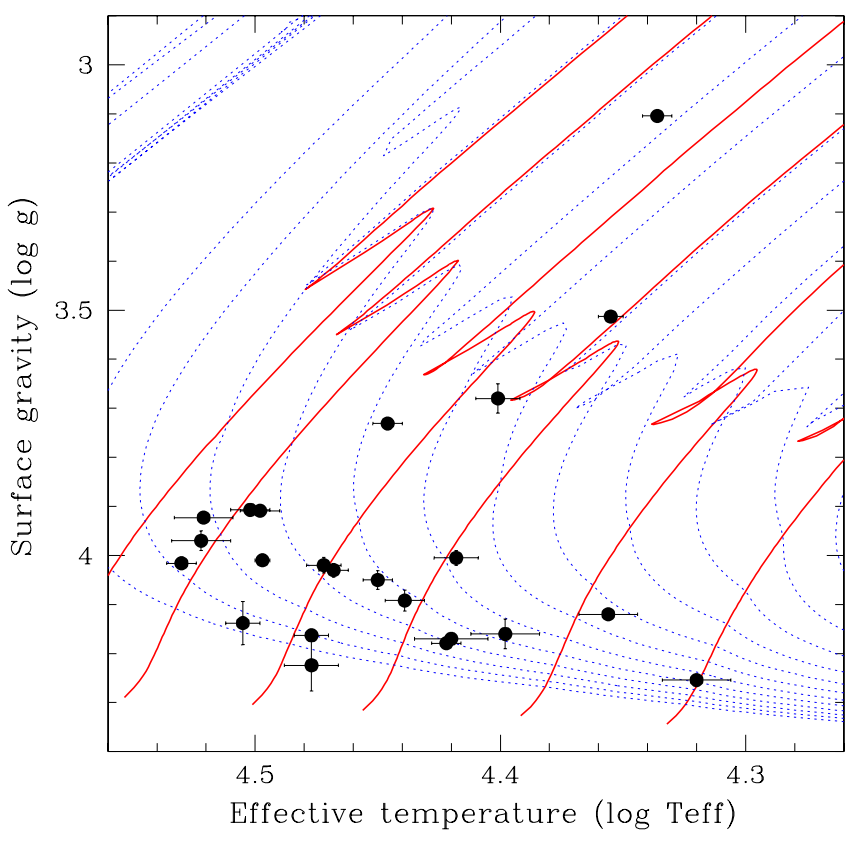

Fig. 1. Location of the stars in our sample of eclipsing binary systems versus the theoretical evolutionary tracks and isochrones from Ekström et al. (2011).

\& Southworth (2009) and Pavlovski et et al. (2009). This scheme makes possible the determination of the fundamental quantities $-M, R, T_{\text {eff }}$ - with the accuracy needed for a useful comparison to theoretical models.

Complementary observables hold an important possibility in the analysis of binary and multiple stars. Only a complementary solution of astrometric and spectroscopic measurements could yield a complete set of the orbital elements and its space orientation. With the recent tremendeous development in interferometric capability, many more spectroscopic binaries have been spatially resolved. The stability and quality of the solution in SPD could be supported with constraints from external observables such as interferometric measurements (Kolbas \& Pavlovski, this proceedings).

An important step in the analysis is determination of the $T_{\text {eff }} \mathrm{S}$ for the stars in binary systems, directly from renormalised disentangled spectra. The traditional degeneracy between $T_{\text {eff }}$ and surface gravity can be lifted for the stars in binary systems. The surface gravities for these star can be determined with uncertainties below 0.01 dex.

A promising and important new development is modelling of the composite spectra for tidally and rotationally distorted stars in binary system given in Palate et al. (2013). 

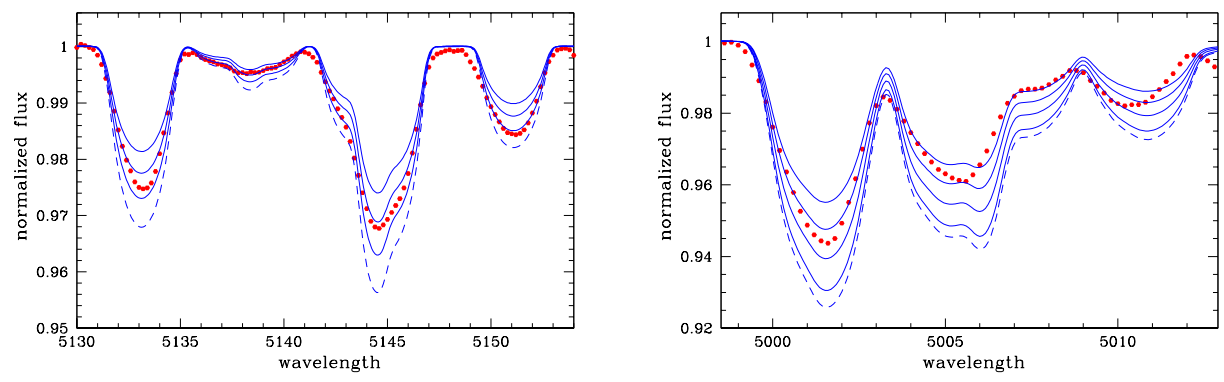

Fig. 2. Comparison between renormalised disentangled spectrum (symbols) of the giant component in V380 Cyg with the synthetic spectra (lines) computed assuming different abundances of C (left panel) and N (right panel). Dashed lines correspond to the 'presentday abundances' as reported by Nieva \& Przybilla (2012).

\section{Abundances for high-mass stars in binary systems}

So far, we have observed and analysed a dozen binary stars with high-mass components. Their location in the Hertzsprung-Russell diagram (HRD) is shown in Fig. 1 along with theoretical evolutionary tracks and isochrones. The majority of the stars are in early phases of their main-sequence evolution, with the youngest systems being V573 Car and V578 Mon. The stars in our sample are late-O to early-B, i.e. at the lower end of the high-mass stars. The largest masses are $20-$ $25 \mathrm{M}_{\odot}$ for the components of V1034 Sco, DN Cas and HD 164258. Their $v \sin i$ values range from $\sim 30 \mathrm{~km} \mathrm{~s}^{-1}$ (V621 Per A; Southworth et al. in preparation) to $250 \mathrm{~km} \mathrm{~s}^{-1}$ (HD 164258 A; Mayer et al. 2013). Of the systems studied, V380 Cyg, V453 Cyg and V621 Per are the most informative as their primary components are evolved to either close to or beyond the terminal-age main sequence (TAMS). Here we described some principal results and summarise published results for the most important systems.

V578 Mon (HD 259135, BD $+4^{\circ} 1299$ ) is an eccentric dEB, in the young open cluster NGC 2244 embedded in the Rosette Nebula, with orbital period $2.408 \mathrm{~d}$. Hensberge et al. (2000) determined the absolute dimensions of the components from uvby photometry and high-resolution spectroscopy. They found the binary to consist of $\mathrm{B} 1 \mathrm{~V}+\mathrm{B} 2 \mathrm{~V}$ stars with masses 14.5 and $10.3 \mathrm{M}_{\odot}$, radii 5.2 and $4.3 \mathrm{R}_{\odot}$, and $v \sin i$ values 114 and $98 \mathrm{~km} \mathrm{~s}^{-1}$. The age inferred from isochrone fitting was estimated to be $\sim 3 \mathrm{Myr}$. Garcia et al. $(2011,2013)$ provided the first measurement of the apsidal motion period and improved the orbital and stellar parameters. A new comprehensive analysis of all available photometric data plus a new échelle spectroscopy with Mercator/HERMES, has allowed a fine-tuning of the physical properties and the internal structure of the stars (Garcia et al. 2014). The photospheric chemical composition of the components has been determined using disentangled spectra (Pavlovski \& Hensberge 2005). Their analysis was done 


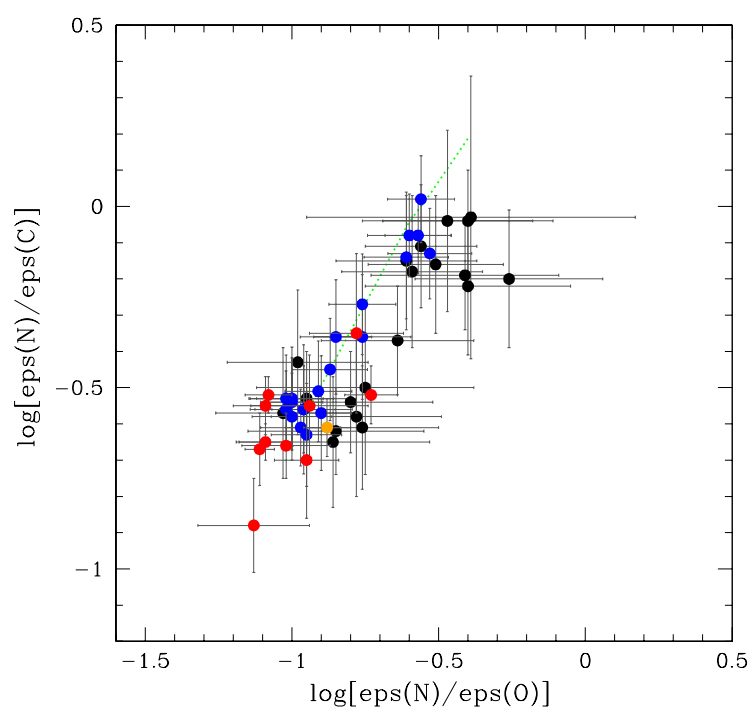

Fig. 3. The trend in $\mathrm{N} / \mathrm{C}$ versus $\mathrm{N} / \mathrm{O}$ ratios found for B stars. Three samples of B stars are shown: single stars from Nieva \& Przybilla (2012) (blue circles), magnetic stars from Morel et al. (2008) (black circles) and stars in close binaries (this work, red circles).

relative to sharp-lined B1 V star \#201 in NGC 2244 for which accurate abundances are known (Vrancken et al. 1997). The abundance pattern for both stars is consistent with those found for several B1 stars in NGC 2244 (Vrancken et al. 1997).

V453 Cyg (HD 227696) is totally eclipsing dEB for which accurate physical properties have been measured (Southworth et al. 2004a). The system consists of two $\mathrm{B}$ stars with masses 14.4 and $11.1 \mathrm{M}_{\odot}$. The more massive component is approaching the TAMS whilst its companion has completed about half of its main-sequence lifetime. These characteristics make V453 Cyg ideal for probing the main-sequence chemical evolution of high-mass stars. The photospheric chemical composition of the components was determined by Pavlovski \& Southworth (2009). The more massive component is the only star in our sample which shows the trend in CNO abundances expected on theoretical grounds (Sect.2, see also Przybilla, this volume). In Fig. 3 the observational results are presented for the three samples: single B stars (Nieva \& Przybilla 2012), single magnetic B stars (Morel et al. 2008) and B stars in close binaries (this work). The trend in the changes of abundance ratio between $\mathrm{N} / \mathrm{O}$ and N/C is governed by the $\mathrm{CNO}$ cycle and stellar evolution (Przybilla et al. 2010; Przybilla, this volume).

V380 Cyg (HD 187879, KIC 5385723) is an eccentric dEB with an evolved Btype component $(\log g=3.1)$. A large discrepancy between the dynamical and evolutionary masses for this component has been found. Guinan et al. (2000) removed 
this mass discrepancy by invoking extremely strong convective core overshooting. Pavlovski et al. (2009) tried unsuccesfully to match the giant component to the rotating evolutionary models of Ekström et al. (2008). As a potential candidate for stochastic pulsations, concerted effort has been put in to observe the binary photometrically with the Kepler satellite, and spectroscopically with Mercator/HERMES (Tkachenko et al. 2012, 2013). This has allowed revision of the absolute dimensions and atmospheric diagnostics for both components even though the secondary contributes only about $6 \%$ of the total light of the system. The measured chemical composition for the giant component corroborates our previous study (Pavlovski et al. 2009). Spectral disentangling of a large collection of high-resolution spectra also makes possible a study of the secondary component. Its composition agrees with the primary's to within the uncertainties. N/C and N/O are characteristic for unevolved B stars, despite one of the component having evolved to the giant stage.

V621 Per $\left(\mathrm{BD}+56^{\circ} 576\right)$ is a totally eclipsing binary system, and a member of the young open cluster $\chi$ Per (NGC 884). The primary star is an early-type subgiant (Southworth et al. 2004b). In an extensive photometric campaign on $\chi$ Per, complete multicolour light curves of this long-period $(P=25.5 \mathrm{~d})$ binary have been obtained (Saesen et al. 2010). An application of spectral disentangling reveals the secondary component which makes possible the determination of the absolute dimensions of the components (Southworth et al. in preparation). It is found that the subgiant component has $M=11.2 \mathrm{M}_{\odot}$, and $\log g=3.5$. Its helpfully low rotation rate $\left(v \sin i=32 \mathrm{~km} \mathrm{~s}^{-1}\right)$, allows an accurate determination of its atmospheric parameters and chemical composition. We find that the nitrogen and carbon abundances do not show the theoretically predicted excess, thus corroborating our results for the giant component of V380 Cyg.

\section{Conclusion}

We have determined the photospheric abundances for helium and CNO elements in high-mass stars in close binaries. We do not find any dependence on their masses, rotational velocities or evolutionary status except for the marginal case of V453 Cyg A. The obvious question is: are high-mass stars in detached binary systems fundamentally different to single high-mass stars for which changes in the photospheric abundances have been disclosed observationally (Hunter et al. 2009, Przybilla et al. 2010, see also Przybilla this volume)? Obviously, tidal effects have some effect on the stellar structure and evolution, and may hinder rotationally induced mixing in high-mass components in close binary systems. This was calculated and discussed by de Mink et al. $(2009,2013)$. However, a significantly larger sample is needed before firm conclusions can be drawn.

\section{References}

Bagnuolo, W. G., \& Gies, D. R. 1991, ApJ, 376, 266

Brott, I., de Mink, S. E., Cantiello, M., et al. 2011, A\&A, 530, A115 
Setting a new standard in the analysis of binary stars

de Mink, S. E., Cantiello, M., Langer, N., et al. 2009, A\&A, 497, 243

de Mink, S. E., Langer, N., Izzard, R. G., Sana, H., \& de Koter, A. 2013, ApJ, 764, 166

Ekström, S., Georgy, C., Eggenberger, P., et al. 2012, A\&A, 537, A146

Frischknecht, U., Hirschi, R., Meynet, G., et al. 2010, A\&A, 522, A39

Garcia, E. V., Stassun, K. G., Hebb, L., et al. 2011, AJ, 142, 27

Garcia, E. V., Stassun, K. G., \& Torres, W. 2013, ApJ, 769, 114

Garcia, E. V., Stassun, K. G., Pavlovski, K., et al. 2014, AJ (submitted)

Guinan, E. F., Ribas, I., Fitzpatrick, E. L., et al. 2000, ApJ, 544, 409

Hadrava, P. 1995, A\&AS, 114, 393

Heger, A., \& Langer, N. 2000, ApJ, 544, 1016

Heger, A., Langer, N., \& Woosley, S. E. 2000, ApJ, 528, 368

Hensberge, H., Pavlovski, K., \& Verschueren, W. 2000, A\&A, 358, 553

Hunter, I., Brott, I., Langer, N., et al. 2009, A\&A, 496, 841

Ilijić, S., Hensberge, H., Pavlovski, K., \& Freyhammer, L. M. 2004, ASP CS, 318, 111

Köhler, K., Borzyszkowski, M., Brott, I., et al. 2012, A\&A, 544, A76

Langer, N. 2012, ARA\&A, 50, 107

Martins, F., Escolano, C., Wade, G. A., et al. 2012, A\&A, 538, A29

Martins, F., Mahy, L., Hillier, D.J., \& Rauw, G. 2012, A\&A, 538, A39

Mayer, P., Harmanec, P., \& Pavlovski, K. 2013, A\&A, 550, A2

Maeder, A., \& Meynet, G. 2012, Review of Modern Physics, 84, 25

Meynet, G., \& Maeder, G. 2000, A\&A, 361, 101

Morel, T., Hubrig, S., \& Briquet, M. 2008, A\&A, 481, 453

Nieva, F. M., \& Przybilla, N. 2007, A\&A

Nieva, F. M., \& Przybilla, N. 2012, A\&A

Palate, M., Rauw, G., Koenigsberger, G., \& Moreno, E. 2013, A\&A, 552, A39

Pavlovski, K., \& Hensberge, H. 2005, A\&A, 439, 309

Pavlovski, K., \& Hensberge, H. 2010, ASP Conf. Ser., 415, 31

Pavlovski, K., \& Southworth, J. 2009, MNRAS, 394, 1519

Pavlovski, K., \& Southworth, J. 2012, IAU Symp., 240, 55

Pavlovski, K., Tamajo, E., Koubský, P., et al. 2009, MNRAS, 400, 791

Pilecki B., Graczyk, D., Pietrzyński, G., et al. 2013, MNRAS, 436, 953

Potter, A. T., Tout, C. A., \& Eldridge, J. J. 2012, MNRAS, 419, 748

Przybilla, N., Firnstein, M., Nieva, M. F., et al. 2010, A\&A, 517, A38

Saesen, S., Carrier, F., Pigulski, A., et al. 2010, A\&A, 515, A16

Sana, H., de Mink, S. E., de Koter, A., et al. 2012, Science, 337, 444

Simon, K. P., \& Sturm, E. 1994, A\&A, 281, 286

Southworth, J., Maxted, P.F.L., \& Smalley, B. 2004a, MNRAS, 351, 1277

Southworth, J., Zucker, S., Maxted, P. F. L., \& Smalley, B. 2004b, MNRAS, 355, 986

Tamajo, E., Pavlovski, K. \& Southworth, J. 2011, A\&A, 526, A76

Torres, G., Andersen, J., \& Giménez, A. 2010, A\&ARev, 18, 67

Tkachenko, A., Aerts, C., Pavlovski, K., et al. 2012, MNRAS, 424, L21

Tkachenko, A., Degroote, P., Aerts, C., et al. 2013, MNRAS (in press), arXiv:1312.3601

Vrancken, M., Hensberge, H., David, M., \& Verschueren, W. 1997, A\&A, 320, 878 